\title{
MICROMECHANICS-DRIVEN VARIATIONAL METHOD FOR DIFFUSE-TO-LOCALISED FRACTURE IN QUASI-BRITTLE SOLIDS
}

\author{
Carlos X. Azua-Gonzalez ${ }^{*}, 1$, Iulia C. Mihai ${ }^{1}$ and Tony Jefferson ${ }^{1}$ \\ ${ }^{1}$ RM4L group, School of Engineering, Cardiff University, UK. \\ Corresponding author*. E-mail addresses: \\ azua-gonzalezcx@cardiff.ac.uk (CAG), mihaiic1@ cardiff.ac.uk (IM), jeffersonad@cardiff.ac.uk (AJ)
}

Key words: Diffuse microcracking to localisation, least-energy approach, strong discontinuities, FEM

\begin{abstract}
A novel multiscale variational method for modelling fracture propagation is proposed. The method employs strong discontinuity kinematics enhancement, enabling macroscopic cracks to be modelled explicitly, while minimum remeshing is ensured. In addition, the response of quadrature points in the bulk is up-scaled using a Micromechanical continua, which enables the evolution of directional micro-defects (e.g. microcracks) without venturing into prohibitive computational burden. Noticeably, the method allows the Micromechanical continua to interact with macroscopic cracks. The framework is conveniently formulated as a variational setting to provide a minimum energy solution. The new computational framework has been found to diagnose realistic failure mechanisms in quasi-brittle materials.
\end{abstract}

\section{INTRODUCTION}

Multiscale homogenisation is resurgent in Computational Mechanics [1], and opens the door to enhance our understanding of materials. Benefits of multiscale modelling are noticeable, when conventional inelasticity frameworks cannot reproduce the response of heterogeneous materials and structures with the required accuracy needed. Taking into account the intrinsic microstructure of materials by direct numerical simulation (DNS) is often computationally prohibitive. On the other hand, computational homogenisation, referred to as global-local analysis [2], can also become computationally demanding [3]. Therefore, there is a need for proposing efficient homogenisation strategies.

Moreover, an adequate or definitive treatment for damage to actual fracture occurrence across scales yet remains as an unresolved dilemma in Multiscale modelling [4]. Although other methods exist for macroscopic fracture, e.g. micropolar continua $[5,6]$, gradient-enhanced continua $[7,8]$, phase-field methods for (quasi-) brittle fracture [9, 10], among others, connection with the evolving microstructure is somewhat hidden in numerical length-scale parameters. Seeking a rigorous micromechanistic basis to macrocracking is appealing. This paper tackles two challenges in Multiscale modelling: (i) reduction of computational cost, e.g. by using diffuse approximations for RVE-based microstructural evolution, and (ii) energetically-consistent treatment of diffuse-micro and localised-macro fracture seamlessly.

The remainder of the paper is divided into six additional sections: Section 2 explains a least-energy approach for multiscale modelling of inelasticity and fracture; Section 3 describes homogenisation principles at the RVE level; Section $\mathbf{4}$ expands on micro to macrocracking transition; Section $\mathbf{5}$ describes the response of sharp macrocracks; Section 6 shows some numerical results; and Section 7 concludes. 


\section{A NEW (LEAST-ENERGY) VARIATIONAL APPROACH TO MICRO-MACRO FRACTURE}

A new variationally consistent method is proposed for modelling the transition from diffuse microcracking to localised fracture in quasi-brittle solids. The method relies on energy minimisation principles, imposed on a multiscale weak form for solids with embedded discontinuities (see figure 2). The reader may refer to $[11,12,13]$ for reference on theoretical perspectives for non-linear solids with embedded discontinuities:

$$
\downarrow \text { Downscaling, } \uparrow \text { Upscaling: } \quad \delta \Pi=\operatorname{minimise}\left\{\int_{\Omega}\left(\delta \varepsilon:<\sigma>_{R V E}\right) d \Omega+\int_{\partial \Gamma^{\mathrm{M}}}\left(\delta[|\mathbf{u}|] \cdot \mathbf{t}_{\Gamma^{\mathrm{M}}}\right) d \Gamma\right\}
$$

$$
\varepsilon \equiv\left(\nabla \mathbf{u}_{c}\right)^{s}=\left(\nabla\left(\mathbf{u}_{c}+\mathscr{H}_{\Gamma^{M}} \otimes[|\mathbf{u}|]\right)\right)^{s}
$$

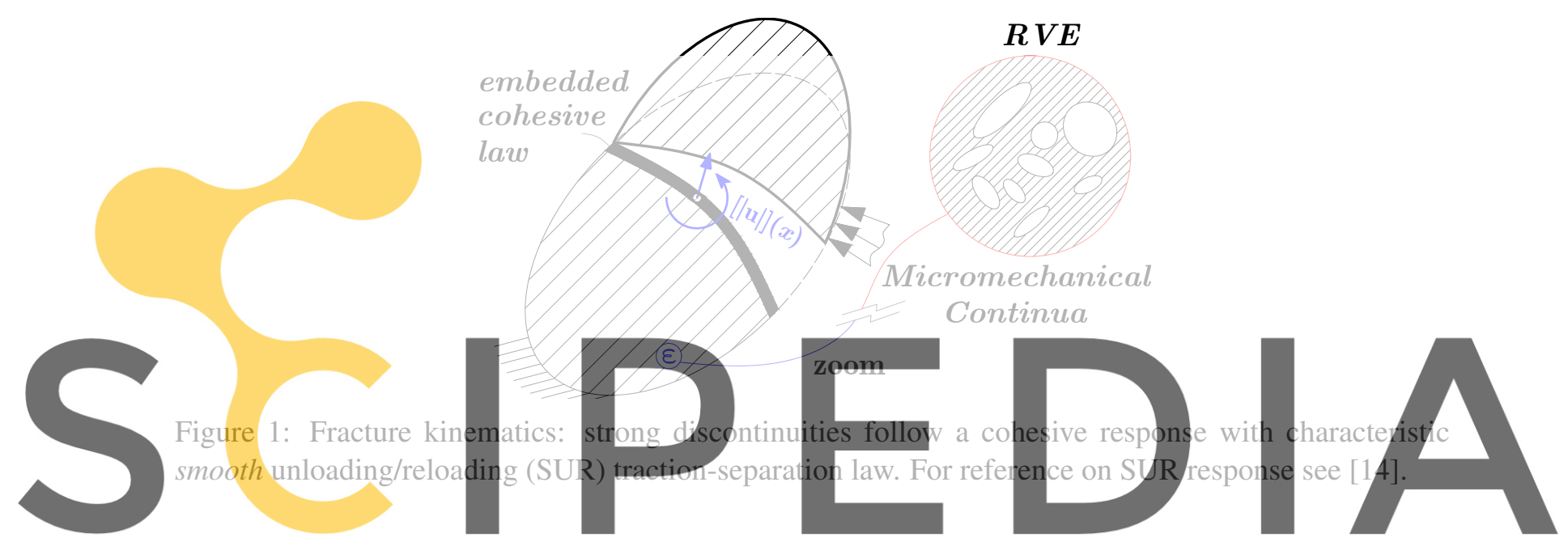

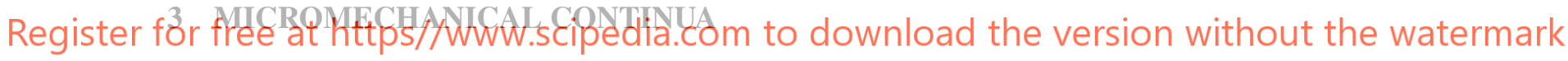

The response of the micromechanical continua is non-linear, and is subjected to the evolution of directional microdefects (e.g. microcracks). Incorporating Eshelby's ellipsoidal micro-voids in a diffuse fashion [15], at various sets of preferential microcracking zones at the RVE level, and employment of volume-averaging rules of RVE-based energy variation (Hill-Mandel condition [16]), yields the following effective constitutive relation:

$$
\begin{gathered}
\varepsilon=<\varepsilon>_{R V E}=D_{e}^{-1}:<\sigma>_{R V E}+\frac{1}{2 \pi} \int_{2 \pi} \int_{\pi / 2} \mathbf{N}_{\varepsilon}^{-1}(\psi, \theta): \varepsilon_{\alpha}^{\Gamma_{m}} \sin (\psi) d \psi d \theta \\
\varepsilon_{\alpha}^{\Gamma_{m}}\left(\varepsilon,<\sigma>_{R V E}\right)=\mathscr{N}_{\alpha} \int_{\partial \Gamma_{m}}\left(r_{\alpha} \otimes\left[\mathbf{u}^{\partial \Gamma_{m}}\right]\right)^{s} d S, \quad \forall x^{\prime} \in \partial \Omega_{\alpha}^{m}
\end{gathered}
$$

Remarks: (i) The evolution of microcrack density in every set of degradation micro-zones is linked conveniently to the average strain history of the RVE, in an implicit computing flow; (ii) Numerical homogenisation, as adopted in this paper, avoids the use of physical domains as RVEs, and exploits energetic principles to track diffuse material degradation at the Micromechanical continuum; (iii) Macrocrack degrees of freedom are condensated at the element level, while micro- and macrocracks interact. 


\section{MACROCRACK DETECTION UPON MICROCRACKS COALESCENCE}

Upon substantial microcracking growth, macroscopic fracture is enabled. In this case, the Micromechanical continua dictates the propagation direction of macroscopic sharp cracks. New macrocracks are allowed to rotate until a critical iteration number itfix is reached. A computational flow is shown below:

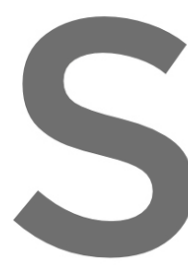

$$
\text { New iterative element displacement (due to recent global update) }: \mathbf{u}^{\mathrm{el}} \leftarrow \mathbf{u}^{\mathrm{el}}+\delta \mathbf{u}^{\mathrm{el}}
$$

$$
\text { Activate flag for macrocrack detection: } f_{\zeta} \leftarrow 1
$$

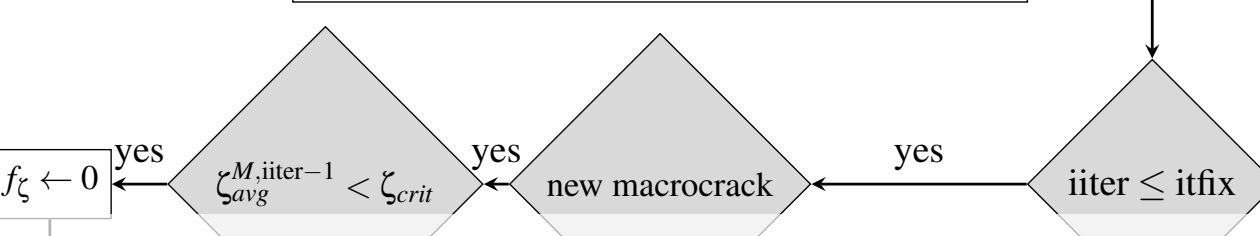

\section{Register for free at https//www.scipedia.com to gdownload the version without the watermark}



Figure 2: Flow chart - Nucleation criteria is based upon crack aperture and Micromechanical stress. 
In addition, new forming macrocracks can be omitted in a near future iteration if the macrocrack average aperture (an equivalent aperture can be used for mixed mode cracks) is smaller than a critical value. This constraint has been found to conveniently delay the release of macrocracks, in such a manner that a smooth transition from micro to macro fracture is obtained in the load-displacement space. A few options are possible to adjust the transition from microcracking dominated phenomena to fully developed macroscopic fracture, although these will be discussed later in the form of a journal publication.

\section{EMBEDDED COHESIVE MODEL FOR SHARP MACROCRACKS}

A simple traction-separation law is used upon macrocrack detection within a localised macrocrack band of defined virtual thickness $h_{c a}$. It is highlighted that there is no geometric thickness of the macrocrack band in the finite element setting. A single degradation macro-state variable $\zeta^{M}$, with units of length, dominates the response at each integration point of the localised macrocrack band. A dimensionless degradation scalar-variable $\omega^{M}$ is defined using a Smooth Unloading Reloading (SUR) approach. Such approach benefits the numerical scheme by ensuring positive tangents upon fixation of macrocracks within the global incremental iterative procedure. The (local) traction separation-law follows the expression shown below :

$$
\mathbf{t}_{\Gamma^{M}}^{\prime}\left(x^{\prime}\right)=\frac{1-\omega^{M}\left(x^{\prime}\right)}{h_{c a}}\left[\begin{array}{cc}
E & 0 \\
0 & E /(2(1+v))
\end{array}\right] \Delta \mathbf{u}\left(x^{\prime}\right), \quad \forall x^{\prime} \in \partial \Gamma^{M}
$$

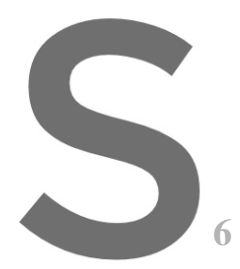

The work-conjugated equivalent force vector for the embedded cohesive model reads as follows:

6 REPRESENTATIVE NUMERICAL EXAMPLE
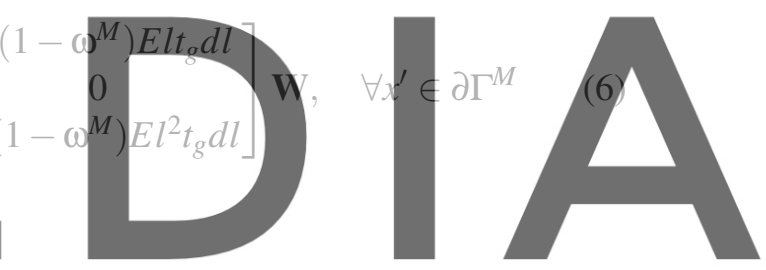

6.1 Four-point bending test

Register for free at https/bwywy-scipe dia,com to downdoad. the version without the watermark

the beam specimen is pushed at two contact points, located at a distance of $L / 3$ from each support axis,

with $L$ being the main span length. For details on element level performance the reader may see [17].

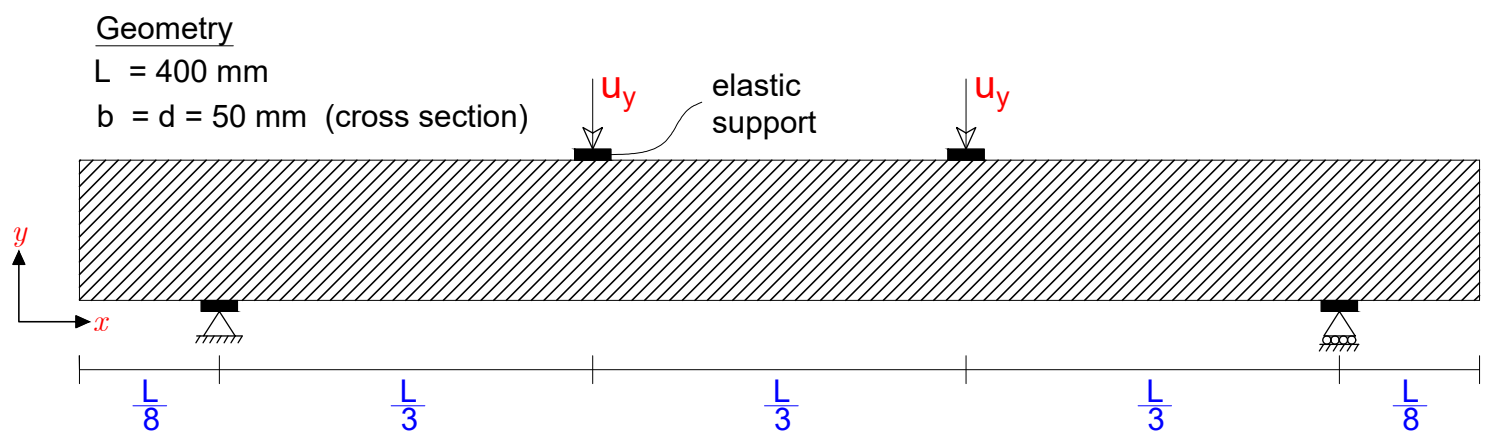

Figure 3: Four point bending test on concrete. Geometry and boundary conditions as shown in illustration. Elastic supports possess equivalent elastic properties as the aggregate-matrix mixture. 
Elastic properties of the aggregate-cement matrix mixture follow standard concrete parameters: overall Young's modulus $E=30 \mathrm{GPa}$ and $v=0.2$. Numerical results are shown below:

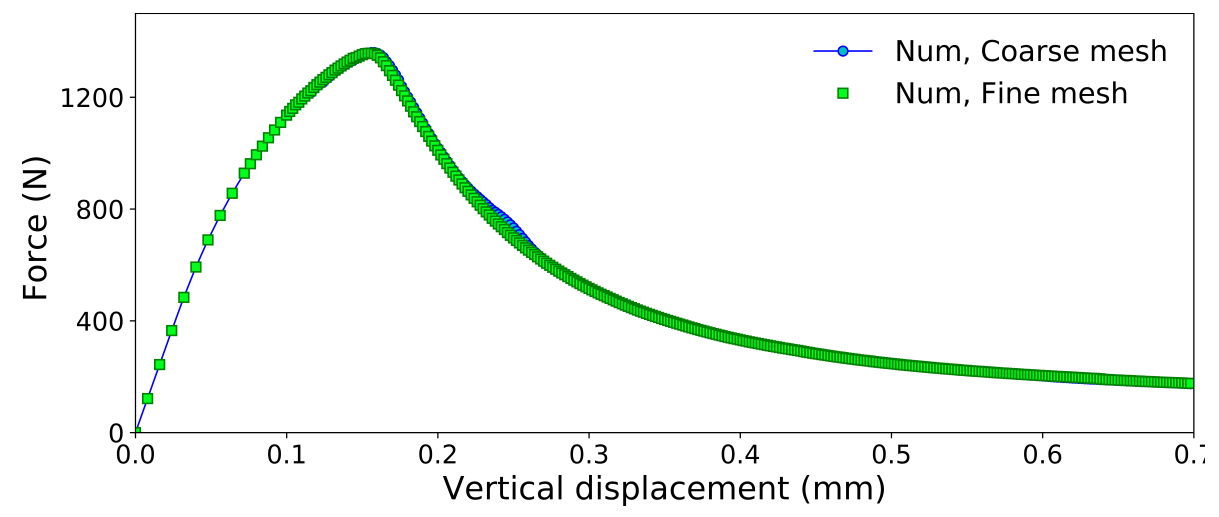

Figure 4: Load-displacement predictions for four-point bending test on concrete.

(a) Coarse mesh (4488 dofs): failure patterns and deformed geometry (shaded background), at $u_{y}=0.2 \mathrm{~mm}$.
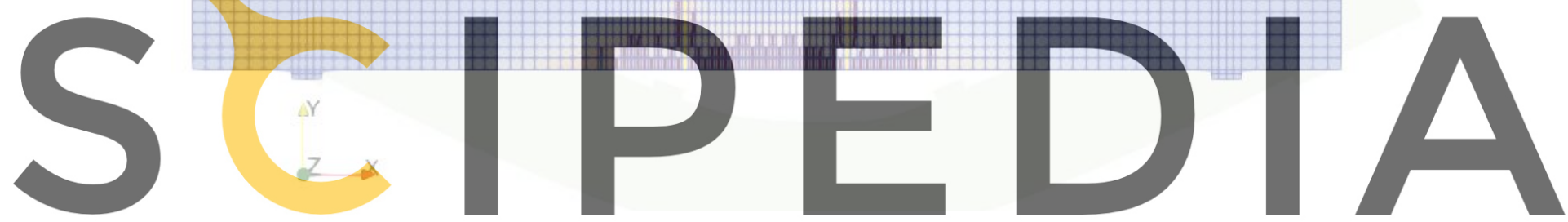

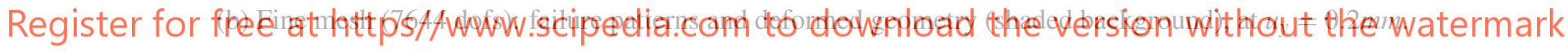
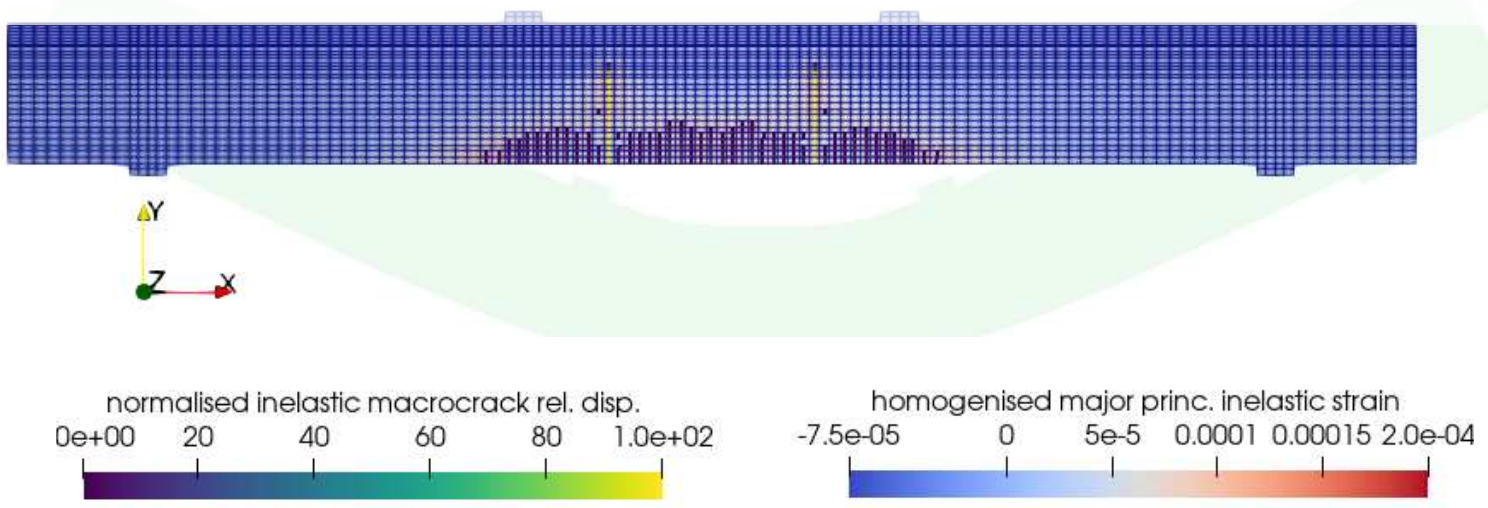

Figure 5: Predictions of failure mechanisms in four-point bending test. Colour-bars indicate inelastic components of (major principal) homogenised strain and normalised macrocrack relative displacement. 


\section{CONCLUSIONS}

A new Multiscale variationally consistent method for modelling fracture, that provides a least-energy solution, is proposed. Distinctive findings read as follows:

- The method is efficient, given that : (i) RVEs are conveniently approximated by a Micromechanical continua; and (ii) macrocracking dofs are condesated at element level.

- The method adequately captures the interaction among diffuse-micro and localised-macro fractures during the onset of material instabilities.

- The method has shown promise in the diagnosis of failure in quasi-brittle materials.

\section{Funding notice and acknowledgment}

Financial support was provided by Cardiff University and the EPSRC-funded grant EP/P02081X/1, awarded to the RM4L project (Resilient Materials for life: https://www.rm41.com). CXAG is thankful to his colleagues Amrit Bains, Brubeck Freeman and Pedro Bonilla for holding regularly inspiring discussions on computational mechanics and inelasticity of cementitious composites.

\section{REFERENCES}

[1] K. Matouš, M. G. Geers, V. G. Kouznetsova, and A. Gillman, "A review of predictive nonlinear theories for multiscale modeling of heterogeneous materials," Journal of Computational Physics,
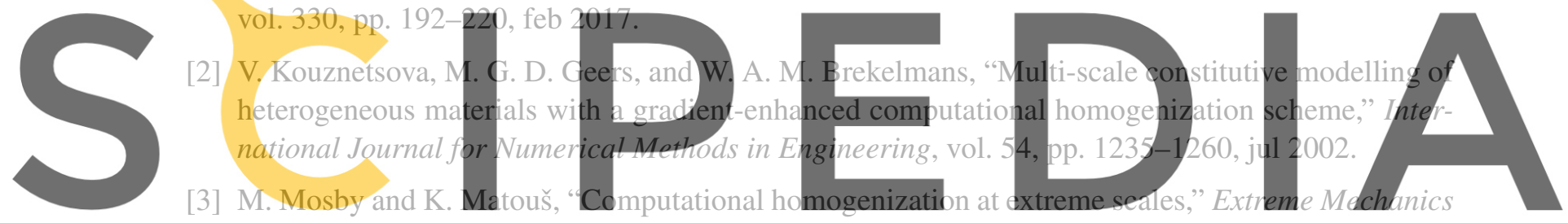
Letters, vol. 6, pp. 68-74, mar 2016.

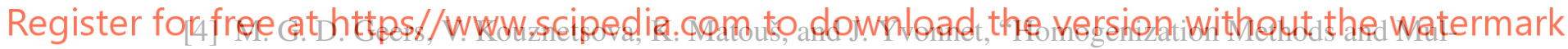
tiscale Modeling: Nonlinear Problems," in Encyclopedia of Computational Mechanics Second Edition. Volume 2: Solids and Structures (E. Stein, R. de Borst, and H. T. J. R., eds.), John Wiley and Sons Ltd, 2017.

[5] R. De Borst, "Simulation of strain localization: a reappraisal of the Cosserat Continuum," Engineering Computations, vol. 8, pp. 317-332, apr 1991.

[6] H. Mühlhaus and I. Vardoulakis, "The thickness of shear bands in granular materials," Géotechnique, vol. 37, pp. 271-283, sep 1987.

[7] R. De Borst and H. B. Mühlhaus, "Gradient-dependent plasticity: Formulation and algorithmic aspects," International Journal for Numerical Methods in Engineering, vol. 35, pp. 521-539, aug 1992.

[8] L. Sluys, R. De Borst, and H. Mühlhaus, "Wave propagation, localization and dispersion in a gradient-dependent medium," International Journal of Solids and Structures, vol. 30, no. 9, pp. 1153-1171, 1993. 
[9] C. Miehe, F. Welschinger, and M. Hofacker, "Thermodynamically consistent phase-field models of fracture: Variational principles and multi-field FE implementations," International Journal for Numerical Methods in Engineering, vol. 83, pp. 1273-1311, sep 2010.

[10] C. V. Verhoosel and R. de Borst, "A phase-field model for cohesive fracture," International Journal for Numerical Methods in Engineering, vol. 96, pp. 43-62, oct 2013.

[11] J. C. Simo, J. Oliver, and F. Armero, "An analysis of strong discontinuities induced by strainsoftening in rate-independent inelastic solids," Computational Mechanics, vol. 12, no. 5, pp. 277296, 1993.

[12] J. Oliver, A. E. Huespe, M. D. G. Pulido, and E. Samaniego, "On the strong discontinuity approach in finite deformation settings," International Journal for Numerical Methods in Engineering, vol. 56, pp. 1051-1082, feb 2003.

[13] F. Armero and C. Linder, "Numerical simulation of dynamic fracture using finite elements with embedded discontinuities," International Journal of Fracture, vol. 160, pp. 119-141, dec 2009.

[14] W. Alnaas and A. Jefferson, "A smooth unloading-reloading approach for the nonlinear finite element analysis of quasi-brittle materials," Engineering Fracture Mechanics, vol. 152, pp. 105-125, feb 2016.

[15] S. Nemat-Nasser and M. Hori, Micromechanics: Overall Properties of Heterogeneous Materials. North-Holland, 1993.

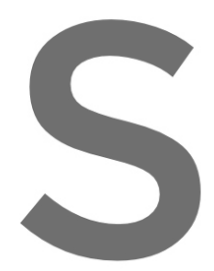

[16] R. Hill, "Elastic chanics and Physics

[17] C. Azua-Gonzalez, approach for Modelling Distributed and Local 19 conference, (London), 2019.
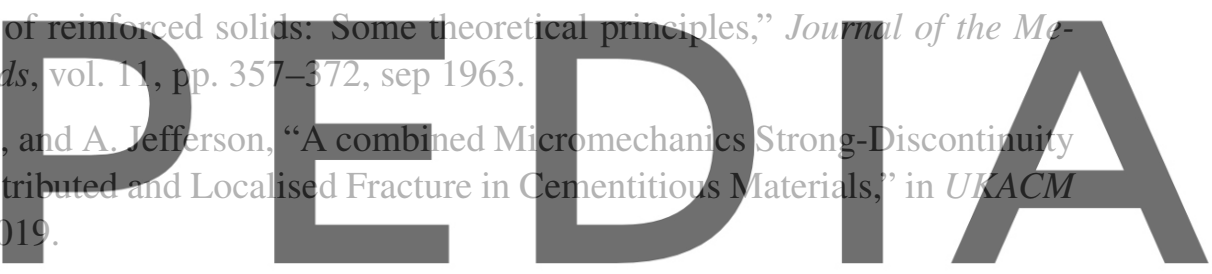

Register for free at https//www.scipedia.com to download the version without the watermark 